\title{
The Herschel view on the dust properties of the Large Magellanic Cloud
}

\author{
Frédéric Galliano \\ AIM, L'Orme des Merisiers, CEA/Saclay, 91191 Gif-sur-Yvette, France
}

\begin{abstract}
I summarize a recent study scrutinizing the various sources of uncertainties in estimating the dust mass of a galaxy. These effects are demonstrated on the Herschel observations of the Large Magellanic Cloud.
\end{abstract}

Keywords. galaxies: ISM — Magellanic Clouds — dust — ISM: evolution

- Motivations. The electromagnetic emission from a galaxy contains information about the physical conditions therein experienced. In star forming regions, most of the power is reradiated by dust, in the infrared. The knowledge of the grain properties (their abundance, chemical composition and size distribution) is therefore crucial to interpret observations of star forming galaxies, nearby and distant. However, the dust properties are known to evolve with their environment, and this evolution is currently poorly constrained. Of particular interest in the Herschel era is the accuracy to which one can estimate the dust mass of a region, and infer its mass of CO-photodissociated molecular gas (so-called "dark gas"). These quantities depend on the accuracy with which we can derive the equilibrium temperature distribution of the grains within our beam, and on the reliability of the assumptions of the long wavelength grain opacities and gas-to-dust mass ratio.

- Method. In a recent study (Galliano et al. 2011), we have explored these problematics by modelling the spatially resolved (down to 10 pc) Spitzer and Herschel broadband observations of the Large Magellanic Cloud (LMC). We have fit the spectral energy distribution (SED) of each pixel from 3 to $500 \mu \mathrm{m}$, with realistic grain properties (silicate, carbon grains and PAHs, with a size distribution) and a distribution of temperatures accounting for the mixing of several regions within our beam. We have rigorously propagated the various sources of errors, accounting for their intercorrelations.

- Results. Our main result is that modelling the LMC with Galactic grain cross-sections leads to violating the elemental abundance constraints. The amount of dark gas is not sufficient to help solve this crisis. We have therefore shown that the intrinsic grain properties of the LMC were systematically different than those of the Galaxy. We have modelled the same data set with an alternate, more emissive, grain composition that is consistent with the elemental depletions.

- Conclusion. We have shown that the assumption of universality of the grain properties does not hold in the LMC. Estimating the dust masses of external galaxies, without additional constraints, is heavily dependent on this assumption. Our study demonstrates that these properties vary among galaxies. In particular, deriving the dust mass of a distant (unresolved) galaxy, in order to estimate its mass of "dark gas", without a "reference" diffuse region, is very speculative.

\section{References}

Galliano, F., Hony, S., Bernard, J.-P., Bot, C., Madden, S. C. et al. 2011, A\&8A, 536, A88 